\title{
LATERALIZAÇÃO DAS FUNÇÕES MUSICAIS NA EPILEPSIA PARCIAL
}

\author{
CLÉO MONTEIRO FRANCA CORREIA*, MAURO MUSZKAT*, \\ NEYDE SANTACHI DE VICENZO*, CARLOS JOSÉ REIS DE CAMPOS*
}

\begin{abstract}
RESUMO - Foram avaliados 14 pacientes destros com idade mediana de 30 anos, portadores de epilepsia parcial (Grupo Epiléptico) e divididos em dois Grupos (Direito e Esquerdo), segundo a lateralização da atividade paroxística no eletrencefalograma interictal. Dos 14 pacientes, $42,8 \%(6 / 14)$ dos casos apresentaram foco à direita, enquanto os $57,2 \%(8 / 14)$ restantes apresentaram foco à esquerda. O Grupo Controle foi formado por 31 indivíduos destros com idade mediana de 30 anos e sem história de doença neurológica ou antecedente de crises epilépticas. Os indivíduos estudados, sem conhecimento musical, realizaram Testes de Habilidades Musicais que envolveram Ritmo Espontâneo, Percepção dos Parâmetros Musicais (timbre, duração, altura, intensidade e ritmo) e Testes Gnósico-Práxicos (reconhecimento e reprodução de parâmetros musicais e organização e reprodução de movimentos corporais rítmicos). Concluímos que a presença do foco no hemisfério cerebral direito afeta o desempenho de funções de reconhecimento melódico, enquanto que nos casos com foco no hemisfério cerebral esquerdo, a reprodução e organização rítmicas estão mais comprometidas quando comparados ao Grupo Controle.
\end{abstract}

PALAVRAS-CHAVE: funções musicais, assimetria funcional hemisférica, epilepsia parcial.

\section{Asymmetry of musical functions in partial epilepsy}

ABSTRACT - Fourteen right handed patients with partial epilepsy (Epileptic Group) and with a median age of 31 years were divided into two groups (Right and Left), according the laterality of paroxystic activity in the eletroencaphalogram. Of the 14 patients, $42.8 \%$ (6/14) presented a focus at the right side while the others $57.2 \%$ (8/14) presented a focus at the left. The Control Group consisted of 31 right handed individuals with a median age of 30 years and with no previous history of neurological disease or epileptic seizures. All the individuals had no musical skills. They carried out Music Abilities Tests including Spontaneous Rhythm, Elemental Music Functions Perception (tone color, duration, pitch, intensity and rhythm) and Complex Strutures Tests (recognition and reproduction of corporal rhythmic movements). We concluded that the focus at the right and the left cerebral hemisphere affect the development of the melodic recognition functions while in cases with focus at the left cerebral hemisphere, rhythmic reproduction and organization are more impaired when compared to the Control Group.

KEY WORDS: musical functions, functional brain asymmetry, partial epilepsy.

Conceituamos "funções musicais" como o conjunto de atividades cognitivas e motoras envolvidas no processamento da música. Tais funções exigem operações mentais multimodais, pois a sua prática envolve a modalidade visual para as notações musicais; a modalidade auditiva para apreciar melodias, ritmos, harmonias e timbres, combinação que define uma peça musical; a expressão motora para a execução musical, que requer a coordenação de diversos músculos e os processos cognitivos e emocionais envolvidos na interpretação da música ${ }^{1}$.

Os distúrbios das funções musicais dificilmente são mencionados nas síndromes neuropsicológicas. No entanto, o desempenho musical pode acrescentar subsídios importantes à avaliação funcional das funções cognitivas. A falta de valorização não se deve à inexistência dos distúrbios, mas à rara utilização de baterias para avaliação dessas funções ${ }^{2-5}$.

* Setor de Investigação e Tratamento das Epilepsias, Disciplina de Neurologia, Universidade Federal de São Paulo - Escola Paulista de Medicina. Aceite: 21-agosto-1998.

Dra. Cléo Monteiro França Correia - Rua Pedro de Toledo 655 - 04039-032 São Paulo SP - Brasil. 
Os métodos convencionais geralmente utilizam a linguagem verbal como veículo das respostas. Porém, o desempenho em atividades musicais pode revestir-se de importância e ser utilizado para complementar a avaliação das funções musicais, uma vez que o processamento cognitivo da música envolve estruturas cerebrais específicas e, muitas vezes, funcionalmente independentes das estruturas envolvidas na linguagem verbal ${ }^{1,6,7}$.

Diversos estudos sobre aspectos relacionados à dominância cerebral surgiram nas últimas décadas, possibilitando, melhor compreensão das funções em relação ao papel dos hemisférios cerebrais e da colaboração funcional entre ambos os hemisférios. A partir desses estudos, tem-se considerado que as habilidades verbais estão especialmente relacionadas com o hemisfério esquerdo, enquanto as habilidades não-verbais dependem principalmente do hemisfério direito ${ }^{8,9}$.

A compreensão das funções musicais tem sido especialmente objeto da pesquisa científica porque estruturas cerebrais específicas estão envolvidas na percepção e realização das atividades musicais ${ }^{1}$.

Os estudos das funções musicais em epilépticos foram basicamente realizados em pacientes com epilepsia de difícil controle, candidatos a cirurgia para a epilepsia. As crises epilépticas põem em evidência o mecanismo de funcionamento das áreas cerebrais, possibilitando o estabelecimento de uma relação entre determinadas alterações do comportamento e funções psíquicas e a localização e a lateralidade do foco ou da lesão. Dentre os poucos trabalhos que estudam o envolvimento das epilepsias e os aspectos musicais, destacam-se os que têm possibilitado o estabelecimento de hipóteses quanto às atribuições dos hemisférios cerebrais em relação a esses aspectos. Assim, nos indivíduos destros atribui-se ao hemisfério esquerdo o julgamento envolvido na percepção do ritmo musical, nos aspectos sequenciais e analíticos da música, duração do som e ordem temporal e ao hemisfério direito, o julgamento na percepção da altura do som, harmonia, timbre, intensidade, melodias e canto $^{10-13}$. No entanto, o desempenho dos músicos, para alguns desses aspectos musicais, não ocorre exatamente da mesma forma pois, por terem passado por um processo de aprendizagem e treinamento musical, podem ter desenvolvido habilidades musicais nos dois hemisférios ${ }^{13-15}$.

A literatura internacional especializada apresenta número reduzido de trabalhos que empregam métodos e recursos técnicos bastante heterogêneos de avaliação das funções musicais e aplicação em indivíduos com diferentes níveis de conhecimento musical e diferentes doenças neurológicas. $\mathrm{O}$ objetivo deste estudo foi o de verificar, através de Testes de Habilidades Musicais realizados em pacientes portadores de epilepsia parcial, as relações entre funções musicais e lateralização de atividade paroxística no eletrencefalograma interictal.

\section{MÉTODOS}

Foi estudado um grupo de 14 pacientes com epilepsia parcial, matriculados no Setor de Investigação e Tratamento das Epilepsias (SITE), da Disciplina de Neurologia da Universidade Federal de São Paulo - Escola Paulista de Medicina (UNIFESP-EPM). As crises epilépticas foram classificadas de acordo com a Classificação Internacional das Crises e Síndromes Epilépticas ${ }^{16-18}$.

Os pacientes do Grupo Estudo e Controle tinham idade variando entre 22 e 50 anos, com idade mediana de 30 anos, de ambos os sexos e com escolaridade mínima de $4^{\text {a }}$ série do Primeiro Grau. Nenhum deles possuía conhecimento musical prévio. Todos foram submetidos a testes auditivos, aos testes de Nível Mental (INV) devendo apresentar desempenho igual ou superior à faixa médio-inferior (QI>79) e aos testes de Conhecimentos Gerais, para garantir a aquisição de conhecimentos fundamentais exigidos no programa de ensino básico formal.

O Grupo Controle (31 casos) constou de 31 indivíduos voluntários, de ambos os sexos, sem história de doença neurológica prévia ou antecedentes de crises epilépticas.

Ao exame eletrencefalográfico intercrítico, os pacientes do Grupo Estudo (Direito e Esquerdo) apresentaram atividade paroxística bem definida e localizada na região temporal anterior, sem difusão ou generalização para o hemisfério contralateral. De acordo com a lateralização da atividade paroxística, o Grupo Estudo foi subdividido em: Grupo Direito ( 6 casos), com atividade paroxística no hemisfério cerebral direito e Grupo Esquerdo ( 8 casos), com atividade paroxística no hemisfério cerebral esquerdo. A epilepsia foi classificada como criptogênica em 7 casos (50\%) e em sintomática nos 7 casos restantes (50\%). Nos casos de epilepsia 
criptogênica, mesmo após investigação clínica, laboratorial e radiológica, não foi possível estabelecer o diagnóstico etiológico. No grupo das epilepsias sintomáticas, a etiologia foi associada a meningite em 2 casos, a traumatismo cranioencefálico em 1 caso, a neurocisticercose em 2 casos, a esclerose mesial hipocampal em 1 caso e a mal formação arteriovenosa em 1 caso. Os pacientes epilépticos eram portadores de epilepsia do lobo temporal de difícil controle e estavam em avaliação pré-cirúrgica. Os exames de tomografia computadorizada do crânio e ressonância nuclear magnética mostraram, no Grupo Epiléptico, normalidade em 7 casos (50\%), enquanto que nos casos restantes foram constatados 3 de calcificação temporal esquerda, 1 de malformação arteriovenosa temporal esquerda, 1 de esclerose hipocampal esquerda e 2 de atrofia córtico-subcortical difusa direita. O critério estabelecido para o nosso estudo foi o de lateralização do foco e não segundo os achados nos exames neuroradiológicos.

\section{Avaliação qualitativa das funções musicais}

A avaliação qualitativa das funções musicais consistiu de uma entrevista, do teste do Ritmo Espontâneo e dos testes musicais.

A entrevista buscou obter informações sobre identificação pessoal, conhecimentos musicais, cultura, ambiente sonoro e história sonora, de forma a serem excluídos os indivíduos com conhecimentos musicais e prática instrumental.

O teste para Avaliação do Ritmo Espontâneo consistiu na avaliação do tempo escolhido espontaneamente por cada indivíduo, para reproduzir, com a mão dominante, uma atividade motora simples (no caso, 21 pulsos).

Os testes musicais compreenderam:

1. Testes para Avaliação da Percepção dos Parâmetros Musicais. Estes, tiveram o objetivo de avaliar a capacidade de percepção auditiva dos parâmetros musicais (duração, timbre, altura, intensidade e ritmo). Para tanto, foram gravadas sequências sonoras para cada parâmetro, de forma que ao ouvi-las, o indivíduo tinha que distinguir os sons fracos dos fortes, o timbre do piano entre os do violino e flauta de Pan, os sons agudos dos graves e os fracos dos fortes. Para a percepção rítmica, foi apresentada uma célula rítmica para ser percebida em uma frase rítmica.

2. Testes para Avaliação das Funções de Reconhecimento e Reprodução de Padrões Musicais e Rítmicos (practo-gnosias musicais). Estes, envolveram os testes de organização e reconhecimento de padrões sonoros, os quais foram subdivididos em:

2.1 Teste de Reconhecimento de Padrões Sonoros Complexos. Com a finalidade de avaliar a capacidade de reconhecer sons de padrão complexo, o examinando foi solicitado a ficar de costas para o examinador, enquanto este produzia os sons, manipulando materiais previamente conhecidos pela população estudada. Assim, o examinador amassou folhas de papel, tocou um sino pequeno, percutiu, com a mão fechada, uma superfície de madeira, deu corda em um relógio despertador e sacudiu um molho de chaves.

2.2 Teste de Reconhecimento e Reprodução de Movimentos Corporais Rítmicos Complexos. Este teste envolveu atividades motoras relacionadas ao esquema corporal. O examinador realizou uma sequência de gestos utilizando partes do corpo: estalos, palmas e palmadas nos joelhos. Com esses movimentos produziu ritmos, obedecendo um determinado número de batidas, devendo, em seguida, ser imitado pelo examinando.

2.3 Teste para o Reconhecimento de Padrão Melódico-Verbal. Este, consistiu na apresentação de pequena frase melódica desconhecida, acompanhada das palavras (letra musical) e gravada em fita cassete, devendo ser reproduzida pelo examinando, logo após a apresentação.

3. Testes para Avaliação das Transposições Musicais: Estes, envolveram atividades que utilizaram, pelo menos, duas vias sensoriais:

3.1 Transposição audio-visual. O teste foi idealizado com o objetivo de avaliar a capacidade do examinando de receber a informação auditiva e reconhecê-la visualmente. Para tanto, utilizou-se uma sequência de 5 sons gravados, conhecidos pela população alvo, e 9 figuras, das quais 5 correspondiam aos sons emitidos. $\mathrm{O}$ examinando foi solicitado a apontar apenas a figura correspondente a cada som ouvido.

3.2 Transposição audio-gráfica. Este, teve a finalidade de avaliar a capacidade de reconhecer os sons e reproduzílos graficamente. Foram utilizados os sons graves e agudos. Em uma folha de papel, foram reproduzidos 5 quadros contendo um número de quadrados (superiores e inferiores) correspondente à quantidade de sequências sonoras emitidas. O examinando tinha que assinalar uma cruz no quadrado superior, quando ouvia o som agudo e uma cruz no quadrado inferior, quando ouvia o som grave.

3.3 Transposição audio-motora: Este teste teve o objetivo de avaliar a capacidade do examinando de reproduzir estruturas rítmicas por imitação, ou seja, de realizar a atividade motora imediatamente após a apresentação do modelo rítmico produzido pelo examinador, obedecendo o andamento e o número de batidas. 
O estudo estatístico dos resultados foi feito mediante a utilização de testes não paramétricos, levando-se em consideração a natureza das variáveis estudadas. Em todos os testes fixou-se o alfa em 0,05 ou 5\% $(\alpha<0,05)$ o nível de rejeição da hipótese de nulidade, assinalando-se com um asterisco (*) os valores significantes.

\section{RESULTADOS}

A Tabela 1 refere-se ao Teste do Ritmo Espontâneo, que mostra o tempo utilizado pelos pacientes epilépticos e indivíduos do Grupo Controle para realizar 21 batidas. Observamos nesse teste que, 12/14 casos epilépticos $(85,7 \%)$ mostraram tendência para realizá-lo com maior rapidez, efetuando as 21 batidas em menos de 10 segundos, enquanto que no grupo controle apenas 16/31 indivíduos $(51,6 \%)$ o fizeram nesse intervalo de tempo. Os dados foram organizados em função da dispersão da mediana 10. Aplicada a Correção de Yates $^{19}$, os resultados deixam de ser estatisticamente significantes, devido ao pequeno número da amostra.

A Tabela 2 mostra os resultados da avaliação do Teste de Reconhecimento de Padrões Sonoros Complexos, obtidos pelos Grupos Epiléptico e Controle, referente ao número de acertos. Nesse teste, o Grupo Epiléptico apresentou menor pontuação (score) do que o Grupo Controle no reconhecimento auditivo de padrões sonoros complexos (ruídos). Os dados foram organizados em função da dispersão da mediana 50.

A Tabela 3, referente ao Teste de Reconhecimento e Reprodução Melódico-verbal, mostra que todos os pacientes do Grupo Direito não conseguiram reproduzir a frase melódico-verbal, enquanto no Grupo Esquerdo apenas 1 paciente foi capaz de realizar o teste.

A Tabela 4, que se refere à Avaliação dos Movimentos Corporais Rítmicos, mostra que na comparação do Grupo Epiléptico com o Grupo Controle apenas os epilépticos com foco no hemisfério cerebral esquerdo (Grupo Esquerdo) tiveram desempenho inferior ao do Grupo Controle.

Tabela 1. Teste do ritmo espontâneo (grupo epiléptico x grupo controle).

\begin{tabular}{lcccc}
\hline Grupos & \multicolumn{1}{c}{ Tempo (segundos) } & Total & Porcentagem \\
& $0-10$ & $>10$ & $(0-\mid 10)$ \\
\hline Epiléptico & 12 & 2 & 14 & 85,7 \\
Controle & 16 & 15 & 31 & 51,6 \\
Total & 28 & 17 & 45 & \\
\hline $\begin{array}{l}\text { Teste do qui-quadrado: } \chi_{\text {calculado }}^{2}=4,77^{*} \\
\text { Correção de Yates }(\mathrm{N}=40)=3,431\end{array}$ & $\chi_{\text {crítico }}^{2}=3,84$ & \\
Valores críticos: $3,841(\alpha=5 \%)$ & $6,635(\alpha=1 \%)$ &
\end{tabular}

Tabela 2. Teste de reconhecimento de padrões sonoros complexos (grupo epiléptico $x$ grupo controle).

\begin{tabular}{lcccc}
\hline Grupo & \multicolumn{2}{c}{ Resposta } & Total & $\begin{array}{c}\text { Porcentagem } \\
(0-\mid 50)\end{array}$ \\
\hline Epiléptico & 6 & 8 & 14 & 42,8 \\
Controle & 1 & 30 & 31 & 3,2 \\
Total & 7 & 38 & 45 & \\
\hline $\begin{array}{l}\text { Teste do qui-quadrado: } \chi_{\text {calculado }}^{2}=11,53 * \\
\text { Correção de Yates }(\mathrm{N}=40)=8,712\end{array}$ & $\chi_{\text {crítico }}^{2}=3,84$ & \\
Valores críticos: $3,841 \quad(\alpha=5 \%)$ & $6,635 \quad(\alpha=1 \%)$ &
\end{tabular}


Tabela 3. Teste melódico-verbal.

\begin{tabular}{lcccc}
\hline Classificação & $\begin{array}{c}\text { Acertaram } \\
\text { o teste } \\
(\mathrm{N})\end{array}$ & $\begin{array}{c}\text { Não } \\
\text { acertaram } \\
(\mathrm{N})\end{array}$ & Total & $\begin{array}{c}\text { \% dos que } \\
\text { acertaram }\end{array}$ \\
\hline Grupo D & 0 & 6 & 6 & 0 \\
Grupo E & 1 & 7 & 8 & 12,5 \\
Grupo C & 16 & 15 & 31 & 51,6 \\
Total & 17 & 28 & 45 & \\
\hline
\end{tabular}

$\mathrm{D}$, foco de projeção direito; $\mathrm{E}$, foco de projeção esquerdo; $\mathrm{C}=$ grupo controle.

Teste do qui-quadrado: $\chi_{\text {calculado }}^{2}=8,34 * \quad \chi_{\text {crítico }}^{2}=5,99$

Participação do qui-quadrado (D) + (E) x (C): $\chi_{\text {calculado }}^{2}=8,11 * \quad \chi_{\text {crítico }}^{2}=3,84$ (G.L.=1)

Tabela 4. Teste de movimentos corporais rítmicos.

\begin{tabular}{lcccc}
\hline Classificação & $\begin{array}{c}\text { Acertaram } \\
\text { o teste } \\
(\mathrm{N})\end{array}$ & $\begin{array}{c}\text { Não } \\
\text { acertaram } \\
(\mathrm{N})\end{array}$ & Total & $\begin{array}{c}\text { \% dos que } \\
\text { acertaram }\end{array}$ \\
\hline Grupo D & 4 & 2 & 6 & 66,6 \\
Grupo E & 0 & 8 & 8 & 0,0 \\
Grupo C & 24 & 7 & 31 & 77,4 \\
Total & 28 & 17 & 45 & \\
\hline
\end{tabular}

$\mathrm{D}$, foco de projeção direito; $\mathrm{E}$, foco de projeção esquerdo; $\mathrm{C}=$ grupo controle.

Teste do qui-quadrado: $\chi_{\text {calculado }}^{2}=16,27^{*} \quad \chi_{\text {crítico }}^{2}=5,99$

Participação do qui-quadrado (E) $\times(\mathrm{D})+(\mathrm{C}): \chi_{\text {calculado }}^{2}=16,02 * \quad \chi_{\text {crítico }}^{2}=3,84(\mathrm{G} . \mathrm{L} .=1)$

Tabela 5. Teste de transposição audio-motora.

\begin{tabular}{|c|c|c|}
\hline Classificação & Acertos por número de pontos & $\overline{\mathrm{X}}$ de pontos \\
\hline Grupo D & $\begin{array}{lllll}13 & 1 & 0 & 4 & 4\end{array}$ & 2,2 \\
\hline Grupo E & $\begin{array}{lllllllll}3 & 0 & 1 & 3 & 0 & 0 & 5 & 1\end{array}$ & 1,6 \\
\hline Grupo C & $\begin{array}{lllllllllllllllll}4 & 2 & 4 & 4 & 1 & 5 & 4 & 4 & 3 & 5 & 1 & 4 & 3 & 5 & 3 & 4 & 4 \\
5 & 4 & 2 & 5 & 0 & 4 & 2 & 5 & 4 & 4 & 0 & 4 & 4 & 3 & & & \end{array}$ & 3,2 \\
\hline
\end{tabular}

$\mathrm{D}$, foco de projeção direito; $\mathrm{E}$, foco de projeção esquerdo; $\mathrm{C}=$ grupo controle. Análise da variância por postos de Kruskal-Wallis

(Grupo Direito x Grupo Esquerdo x Grupo Controle): $\mathrm{H}$ calculado $=7,5^{*} \quad \mathrm{H}$ crítico $=5,99$

Teste de comparações múltiplas: Grupo Esquerdo < Grupo Controle.

A Tabela 5, referente ao Teste para Avaliação da Transposição Audio-Motora, mostra que o Grupo Epiléptico com foco temporal à esquerda (Grupo Esquerdo) teve um desempenho inferior ao do Grupo Controle.

\section{DISCUSSÃO}

Para este estudo, houve uma constante preocupação em compor um grupo homogêneo, pois a interferência de variáveis como sexo, idade, dominância lateral manual, síndromes neurológicas diversas ${ }^{20,21}$ e diferentes níveis de conhecimentos musicais ${ }^{13,22}$ podem criar dificuldades metodológicas 
na comparação dos resultados. Além disso, foi difícil compor os Grupos Epiléptico e Controle, pois ambos teriam que apresentar características semelhantes em relação à dominância lateral manual, quociente de inteligência, níveis de escolaridade e de conhecimentos gerais, acuidade auditiva e ambiente sonoro.

A opção de avaliar qualitativamente as funções musicais se deve à possibilidade de oferecer uma contribuição aos testes de avaliação de funções cognitivas convencionais, os quais valorizam a linguagem verbal, como veículo das respostas.

No Teste do Ritmo Espontâneo, os pacientes do Grupo Epiléptico (com foco nos hemisférios cerebrais direito e esquerdo) apresentaram tendência a apresentar um "ritmo espontâneo" mais rápido que o do Grupo Controle (Tabela 1). No entanto, com a Correção de Yates, o nosso resultado deixa de ser estatisticamente significante, podendo atribuir esse fato ao pequeno tamanho da amostra.

Segundo Mira Stambak ${ }^{23}$, idealizadora do teste, o ritmo espontâneo traduz um padrão rítmico individual que não apresenta grande variabilidade após os 12 anos de idade. As oscilações das batidas podem estar relacionadas a modificação do ritmo espontâneo, que pode alcançar maior estabilidade a partir dessa faixa etária.

Com referência aos aspectos gnósico-práxicos, observamos que a literatura consultada para a busca de conceitos sobre gnosia e praxia nos mostrou que os autores se detiveram na conceituação dos distúrbios agnosia e apraxia, separadamente. No entanto, Barbizet \& Duizabo ${ }^{24}$ consideram-nas conjuntamente, como resultado de uma integração sensório-motora, preferindo o termo apractognosia para designar as desorganizações das habilidades adquiridas de todas as espécies, ou seja, as que se referem ao conhecimento que cada indivíduo tem de seu próprio corpo, das relações estabelecidas entre este e os objetos à sua volta e com o espaço onde se move. Assim, falam na desorganização do "saber-fazer", tratando-se de distúrbios decorrentes de comprometimento das zonas associativas têmporo-parieto-occipitais de ambos os hemisférios, na ocorrência de lesões. Nesses casos, considerou-se que existe uma participação maior do hemisfério direito no desempenho dessas atividades.

Assim, neste estudo, preferimos considerar os aspectos gnósico-práxicos em conjunto, para facilitar a compreensão dos nossos achados.

Os aspectos gnósico-práxicos envolveram:

a) Reconhecimento de padrões sonoros complexos:

Quando nos referimos aos aspectos essencialmente gnósicos, podemos dizer que um indivíduo normal tem capacidade para reconhecer a maioria dos ruídos que o envolve e esse reconhecimento, em condições normais de vida, pode ser auxiliado pelo contexto, ou seja, por informações sensoriais associadas, ou então pode ocorrer pelo fato do ruído fazer parte do cotidiano do indivíduo. Foi o que observamos em relação ao Grupo Controle, cujos indivíduos não tiveram dificuldade para realizar essa atividade.

Os nossos resultados mostram que tanto os pacientes do Grupo Esquerdo como os do Direito apresentaram maior dificuldade para o reconhecimento desses sons, em relação ao Grupo Controle (Tabela 2). Tais dificuldades podem ter sido devidas a disfunções em áreas cerebrais associativas, como a região temporo-límbica, que podem determinar dificuldades tanto na identificação e diferenciação de estímulos sonoros como em sua associação com imagens representativas desses sons (figuras).

O processo para identificação desses ruídos é complexo e os dois hemisférios cerebrais não estão igualmente envolvidos, segundo a natureza do estímulo ${ }^{4,25,26}$.

Preferimos, neste estudo, utilizar sons de padrões complexos considerados familiares para qualquer indivíduo. No entanto, tal escolha pode ter influenciado nossos resultados, uma vez que um estímulo familiar é geralmente processado pelos dois hemisférios cerebrais ${ }^{4}$.

Quando o padrão sonoro é novo e complexo, a sua análise e identificação passa a ser mediada pelo hemisfério não-dominante (geralmente o direito em destros), mas quando repetido, passa a ter 
um valor simbólico tornando-se similar a uma mensagem significante, compreendida e denominada pelo hemisfério esquerdo como se fosse familiar. A sua representação cerebral será efetivada ao nível dos dois hemisférios cerebrais, que intervirão no seu reconhecimento e verbalização ${ }^{24}$.

\section{b) Reconhecimento e reprodução Melódico-Verbal}

Os pacientes epiléticos (Grupos Direito e Esquerdo) tiveram desempenho inferior ao do Grupo Controle no Teste para Avaliação do Reconhecimento e Reprodução Melódico-Verbal (Tabela 3).

A investigação da capacidade de ouvir um trecho melódico, memorizar e reproduzi-lo é importante para o estudo da memória não-verbal, intimamente relacionada à integridade de regiões temporais, habitualmente do hemisfério não dominante e das regiões pré-motoras do córtex cerebral. Dificuldades na memorização de melodias podem constituir a única manifestação neuropsicológica associada à lesão temporal direita ${ }^{4}$.

Devido à grande variabilidade de aptidões musicais nos seres humanos, o estudo do reconhecimento melódico e sua reprodução deve ser conduzido através de testes simples, pois os mais complexos deverão ser apresentados apenas para indivíduos que desenvolveram habilidades musicais, por apresentarem diferentes estratégias no processamento desses aspectos ${ }^{4,13,27-29}$. O nosso teste consistiu de uma frase melódico-verbal desconhecida, a qual permitiu que os indivíduos não músicos fossem capazes de entoá-la, como aconteceu com o Grupo Controle.

Foi interessante observar que os pacientes epilépticos não apresentaram dificuldades para discriminar os parâmetros sonoros quando avaliados nos testes perceptivos, separadamente, pois o desempenho perceptivo desses parâmetros pode envolver áreas cerebrais mais restritas, relacionadas às áreas de projeção primária do córtex auditivo. No entanto, quando apresentados conjuntamente, em um contexto melódico, as dificuldades tornaram-se mais evidentes. Podemos sugerir que, a partir do momento em que esses indivíduos precisaram recrutar áreas cerebrais (plurimodais) responsáveis por aspectos mais complexos do processamento sonoro, começaram a surgir as diferenças entre o Grupo Epiléptico e o Controle.

De acordo com os nossos achados, podemos sugerir que as diferenças para a reprodução oral dos sons apresentados podem ser consequência de alterações na análise e síntese sonoras, decorrentes de lesão ou disfunção nos setores têmporo-límbicos do cérebro ou de distúrbios relacionados ao ato motor de cantar'.

Alguns estudos têm mostrado que os efeitos de lateralidade dependem não apenas da natureza do estímulo, mas também, das estratégias ou modos de processamento utilizado pelos indivíduos. Bever \& Chiarello ${ }^{13}$ mostraram que os indivíduos que tocavam algum instrumento por cerca de 4 anos, reconheciam melhor as melodias pelo hemisfério cerebral esquerdo do que pelo direito, enquanto não músicos apresentaram efeitos opostos. Peretz \& Morais ${ }^{30}$ sugerem que somente os músicos têm condições de processar melodias de forma analítica (propriedade do hemisfério esquerdo) e não músicos, de processar de forma global (ou holística), que é típico do hemisfério direito. Portanto, a forma de processar depende da experiência musical.

Os nossos achados sugerem um papel preponderante das regiões temporais dos dois hemisférios cerebrais no reconhecimento e reprodução de melodias, uma vez que tanto o Grupo Epiléptico Direito como o Grupo Epiléptico Esquerdo apresentaram maior dificuldade no desempenho do teste.

\section{c) Movimentos corporais rítmicos complexos - Grupo Esquerdo}

No Teste para Avaliação dos Movimentos Corporais Rítmicos, os pacientes epilépticos, com foco de projeção temporal esquerda, tiveram desempenho inferior ao do Grupo Controle, mas não em relação ao Grupo com foco de projeção temporal direita (Tabela 4). No nosso estudo, o Grupo Controle pôde reproduzir os gestos imediatamente após o examinador, tendo mostrado boa capacidade de observação dos diversos movimentos apresentados e boa referência em relação ao seu próprio corpo, além de terem apresentado boa capacidade para reter as informações visual e auditiva, organizar e reproduzir, em sequência, os movimentos corporais.

Os gestos intencionais parecem não se resumir apenas em atividades motoras, mas são o resultado de um longo aprendizado que se acumula sob a forma de engramas na região têmporo- 
parieto-occipital ${ }^{4,24}$. Os gestos mais complexos podem requerer a realização de atos motores sequenciais que culminam com o objetivo a ser atingido.

Os pacientes epilépticos pertencentes ao Grupo Direito também conseguiram realizar as atividades, estando de acordo com o conhecimento de que o hemisfério cerebral direito não está diretamente envolvido com os aspectos rítmicos e de organização dos movimentos corporais ${ }^{26}$.

Estudos em afásicos e em pacientes com distúrbios vísuo-espaciais e perturbação do esquema corporal mostram que os movimentos corporais rítmicos complexos podem parecer alterados na ocorrência de lesões da área têmporo-parieto-occipital esquerda, em decorrência de lesões do hemisfério esquerdo ${ }^{24}$.

Portanto, se considerarmos que o hemisfério esquerdo está mais relacionado com o planejamento motor e com a sequenciação temporal de estímulos ${ }^{26-28}$, provavelmente teremos explicado o fato dos pacientes epilépticos, pertencentes ao Grupo Esquerdo, não terem conseguido realizar a atividade de movimentos corporais rítmicos. O seu desempenho caracterizou-se por vacilação na sequência dos gestos, além de erros no número de batidas de palmas, estalos ou palmadas nos joelhos.

\section{d) Reprodução de estruturas rítmicas}

Entendemos por transposição gnósica a recepção da informação por uma via sensorial e a realização da atividade por outra ${ }^{26}$. Assim, a avaliação das possibilidades de transposição deve fazer parte da investigação das coordenações audiomotoras, ou dos atos motores, pela sua importância para o diagnóstico das lesões focais no córtex cerebral ${ }^{4}$. Em vista disso, o teste de reprodução de estruturas rítmicas reveste-se de importância, por envolverem áreas cerebrais responsáveis pela captação auditiva dos estímulos e organização sequencial dos movimentos.

No Teste de Reprodução de Estruturas Rítmicas (Transposição), os pacientes com foco temporal esquerdo tiveram desempenho inferior ao do Grupo Controle (Tabela 5).

Indivíduos neurologicamente normais, ao serem submetidos ao teste de reprodução rítmica realizam-no sem dificuldades, assim como os pacientes com lesões focais fora das áreas temporais e fronto-temporais ${ }^{4}$. Se o indivíduo puder perceber as estruturas rítmicas auditivamente, mas não puder realizar os movimentos por alteração na organização consecutiva dos atos motores, a realização da prova estará prejudicada. Os pacientes que apresentam lesão no córtex do lobo temporal, principalmente à esquerda, poderão apresentar dificuldades na realização desta prova ${ }^{4}$.

Dentre os testes para avaliação da transposição audiomotora aos quais os nossos pacientes epilépticos foram submetidos, o teste de reprodução de estruturas rítmicas foi o que trouxe dificuldades para os pacientes pertencentes ao Grupo Esquerdo. Estes não conseguiram realizar a atividade.

A exemplo do Teste de Organização dos Ritmos Corporais, o Grupo Direito teve um bom desempenho, sugerindo que o hemisfério direito não é o preponderante para o desempenho de aspectos rítmicos e de sequenciação dos movimentos corporais. Não encontramos estudos sobre habilidades rítmica e melódica em pacientes portadores de epilepsia não-lesional, o que dificulta a abrangência e análise de nossos achados em relação à própria epilepsia.

Distúrbios das funções musicais, principalmente referindo-se aos aspectos ritmo e melodia, têm sido observados quase que exclusivamente em pacientes afásicos, portadores de lesões cerebrais lateralizadas e extensas ${ }^{5,31-33}$. As habilidades musicais podem permanecer preservadas na ocorrência de distúrbios da linguagem e, portanto, a existência de dissociação entre distúrbios musicais e verbais sugere uma independência funcional relativa de substratos neurobiológicos para cada atividade ${ }^{1,3,7}$.

Estudos posteriores poderão ser realizados com o objetivo de isolar o efeito lesão e o efeito atividade paroxística, pois a lesão pode produzir um efeito oposto sobre a lateralização hemisférica das funções cognitiva e perceptiva ${ }^{34}$.

Os testes propostos neste estudo, por envolverem áreas cerebrais distintas em relação às da linguagem, permitem detectar déficits que não dependem de áreas relacionadas à linguagem verbal para a sua realização. Consideramos, portanto, que podem constituir um instrumento adicional de diagnóstico neuropsicológico em pacientes portadores de epilepsia parcial, permitindo acessar diferentes áreas cerebrais envolvidas com o processamento sonoro das informações sensoriais auditivas. 


\section{REFERÊNCIAS}

1. Sergent J. Music, the brain and Ravel. TINS 1993;16:168-172.

2. Allegri R. Estudio de las funciones musicales en los pacientes afasicos. Musicoterapia 1991;2:34-39.

3. Huber W, Poeck K, Willmes K. The Aachen Aphasia Test. Adv Neurol 1984;42:292-303.

4. Luria AR. Las funciones corticales superiores del hombre. 2.Ed. Habana: Ministerio de Cultura Editorial CientificoTécnica, 1982.

5. Selinger M, Prescott TE. Auditory event-related potential probes and behavioral measures of aphasia. Brain \& Language 1989;36:377-390.

6. Hachinski KV, Hachinski V. Music and the brain. Can Med Assoc J 1994;151:293-296.

7. Sergent J, Zuck E, Terriah S, MacDonald B. Distributed neural network underlying musical sight-reading and keyboard performance. Science 1992;257:106-109.

8. Brodal A. Sistema Auditivo. In Brodal A (ed). Anatomia neurológica com correlações clínicas. São Paulo: Roca, 1984.

9. Kimura, D. Some effects of temporal lobe damage on auditory perception. Can J Psychol 1961;15:157-165.

10. Bradshaw JL, Nettleton NC. Human cerebral asymmetry. New Jersey: Prentice-Hall, 1983.

11. Gates A, Bradshaw JL. Music perception and cerebral asymmetries. Cortex 1977;13:390-404.

12. Taylor, D. A neuroanatomical model for the use of music in the remediation of aphasic disorders. In Lee MHM (ed). Rehabilitation, music and human well-being. St. Louis: M.M.B. Music, 1989:168-178.

13. Bever TG, Chiarello RJ. Cerebral dominance in musicians and non-musicians. Science 1974;185:537-539.

14. Despins JP. La musica y el cerebro. Barcelona: Editora Gedisa, 1989.

15. Springer SP, Deutsch G. Left brain, right brain. New York: W.H. Freeman \& Co, 1985.

16. Comission on Classification and Terminology of the International League Against Epilepsy. Proposal for revised clinical and eletroencephalographic classification of epileptic seizures. Epilepsia 1981;22:489-501.

17. Comission on Classification and Terminology of the International League Against Epilepsy. Proposal for classification of epilepsies and epileptic syndromes. Epilepsia 1985;23:268-278.

18. Comission on Classification and Terminology of the International League Against Epilepsy. Proposal for revised classification of epilepsies and epileptic syndromes. Epilepsia 1989;30:389-399.

19. Siegel S. Estadistica no parametrica. México: Trillas, 1975.

20. Mazzucchi A, Parma M, Cattelani R. Hemispheric dominance in the perception of tonal sequences in relation to sex, musical competence and handedness. Cortex 1981;17:291-302.

21. Muszkat M, Frayman L, Vincenzo NS, Campos CJR. Diferenças sexuais na assimetria funcional hemisférica: avaliação taquistoscópica verbal utilizando microcomputador. Arq Neuropsiquiatr 1991;49:142-146.

22. Kester DB, Saykin A, Sperling MR, O'Connor MJ, Robinson LJ, Gur RC. Acute effect of anterior lobectomy on musical processing. Neuropsychologia 1991;29:703-708.

23. Stamback, M. Tempo Espontâneo. In Zazzo R (ed). Manual para o exame psicológico da criança. São Paulo: Mestre Jou, 1968:107-125.

24. Barbizet J, Duizabo P. Manual de neuropsicologia. São Paulo: Masson do Brasil, 1985.

25. Spellacy F. Lateral preferences in the identification of patterned stimuli. J Acoust Soc Am 1970;47:574-578.

26. O'Boyle MW, Sanford M. Hemispheric asymmetry in the matching of melodies to rhythm sequences tapped in the right and left palms. Cortex 1988;24:211-221.

27. Burton A, Morton N, Abbes S. Mode of processing and hemisphere differences in the judgement of musical stimuli. Br J Psychol 1989;80:169-180.

28. Mesulam MM. Principles of behavioral neurology. Philadelphia F.A. Davis, 1985.

29. Johnson O, Kozma A. Effects of concurrent verbal and musical tasks on a unimanual skill. Cortex 1977;13:11-16.

30. Peretz I, Morais J. Modes of processing melodies and ear asymmetry in no musicians. Neuropsychologia 1980;18:477-489.

31. Yamadori A, Osumi Y, Masuhara S, Okubo M. Preservation of singing in Broca's aphasia. J Neurol Neurosurg Psychiatry 1977;40:221-224.

32. Brust JCM. Music and language: music alexia and agraphia. Brain 1980;103:367-392.

33. Basso A, Capitani E. Spared musical abilities in a conductor with global aphasia and ideomotor apraxia. J Neurol Neurosurg Psychiatry 1985;48:407-412.

34. Muszkat M, Vincenzo NS, Reami DO, Almeida CIR, Campos MI, Campos CJR. Hemispheric specialization in partial epilepsy. Arq Neuropsiquiatr 1991;49:384-391. 